\title{
On control design for a lower limb orthosis: A comparative study in different operating conditions
}

\author{
N.Roula \\ LPA, Fanar, Lebanon \\ Lebanese University \\ Faculty of Sciences
}

\author{
A.Chemori \\ LIRMM, 161 Rue Ada \\ University of Montpellier 2 \\ Montpellier, France
}

\author{
R.Rizk \\ CRSI, Roumieh, Lebanon \\ Lebanese University \\ Faculty of engineering
}

\author{
Y.Zaatar \\ LPA, Fanar, Lebanon \\ Lebanese University \\ Faculty of Sciences
}

\begin{abstract}
This paper deals with the control of a lower limb orthosis. In which a model of the shank-orthosis system is given, we consider the human effort as external torque acting on the system. A comparative study, through a number of simulations in different operational scenarios, highlights the limits of a standard PID controller. On another hand, this work brings out the benefits of orienting the control strategies toward model reference approaches. Hence, a better exploitation of the nonlinear system dynamics; by deriving adaptive control strategies to enable the management of parameters uncertainty could be a relevant approach for such systems

Keywords-Orthosis, Exoskeleton, Adaptive control, PID, Sliding mode.
\end{abstract}

\section{INTRODUCTION}

Nowadays Robotics is presented as a solution to the rehabilitation and assistance of dependent people. Wearable robots are designed to fit human anatomies. They have to ensure the best assistance to people having physical weaknesses, in their daily tasks. Also, wearable robots are used to ensure the physical and neurological rehabilitation of joints and muscles. They lead patients to recover and regain their mobility. Three case studies are considered in this paper: Passive rehabilitation concerns people who have lost control of their limbs. The second case is the assistance as needed where the wearer is involved to develop a muscular torque in the same direction as the desired trajectory. The third case is the resistive rehabilitation where the patient is asked to develop an effort in the opposite direction of the robots movement [1] [2] [3]. The intention of the subject can be estimated using EMG electrodes fixed at the thigh muscles. [4]. Maximizing the transparency of the system to the wearer, particularly in an assistance as needed and a resistive rehabilitation contexts is one of the main problems addressed. Changes in the humanorthosis dynamics during the rehabilitation processes need to be efficiently compensated. A classical PID (proportional, integral, derivative) structure for gait assistance was designed. One of its derivatives: the proportional controller was tested. This controller amplifies the human torque measured by EMG. [5] [6]. The proportional derivative controller (PD) was also developed in [7] and shoes insoles were used to measure the human fore. In [8], a model reference adaptive control was developed. This controller was designed in order to compensate for dynamics changes in human-orthosis system [9] [10] [11]. This technique has minimized the torque driving the system to the reference model. The control law is based on an on-line modulation of the control parameters. The writers highlighted the main advantage in using adaptive strategy which is the online parameters computation to ensure the best convergence of the trajectory. This control law is subject of a study in this paper. In [12] augmented $\mathcal{L} 1$ adaptive control is developed and tested. An additional nonlinear proportional controller term is added to the torque input. The main aim of this proposed law is to mitigate the drawback due to the time lag introduced by a classical $\mathcal{L} 1$ adaptive law. The paper is structured as follows. In section II, the shank-foot-exoskeleton model is presented based on previous modeling works. In section III the three control laws: PID, Sliding Mode and a Model Reference adaptive control are presented with the stability studies related to each one. Simulation results are addressed in section IV for five scenarios. Finally, conclusions and future works are given in section V.

\section{SysteM's Modeling}

EICOSI (cf. Fig. 1) is a one DOF lower limb exoskeleton. A brushless DC motor drives the wearable robots and an incremental encoder measures the knee joint angle. When embodied by the wearer and attached to his leg using straps the whole system shank-foot-exoskeleton rotates synchronously and is considered as an entity that is put in movement when one (The control Torque $\tau$ ) or two torques (The control Torque $\tau$ and the Human torque $\tau_{h}$ ) are applied to the system as external inputs. $J_{s}, \tau_{g s}, A_{s}, B_{s}$ and $K_{s}$ are respectively the shank inertia, gravity torque, solid friction coefficient, viscous friction coefficient and the stiffness coefficient. $J_{0}, \tau_{g 0}, A_{0}, B_{0}$ and $K_{0}$ are respectively the orthosis inertia, gravity torque, solid friction coefficient, viscous friction coefficient and the stiffness coefficient. We consider the subject in a seated position, and the shank is hanging without any contact with the floor. Flexion and extension movements of the knee joint are studied in this work. The system tries to track a desired trajectory [13]. By deriving the Lagrangian of the shank-footexoskeleton system, the rotational dynamics can be obtained:

$$
J \ddot{\theta}=-\tau_{g} \cos \theta-A \operatorname{Sign} \dot{\theta}-B \dot{\theta}-K\left(\theta-\theta_{\text {rest }}\right)+\tau+\tau_{h}
$$

With

$$
\begin{gathered}
J=J_{s}+J_{0}, A=A_{s}+A_{0}, B=B_{s}+B_{0}, \\
\tau_{g}=\tau_{g s}+\tau_{g 0}, K=K_{s}+K_{0} .
\end{gathered}
$$

$\theta$ is the knee joint angle between the actual position of the shank and the full extension position, $\dot{\theta}$ and $\ddot{\theta}$ are respectively the knee joint angular velocity and acceleration, $\theta_{\text {rest }}$ is the rest position of the shank. Sign () is a signum function, $\tau$ is the control torque and $\tau_{h}$ is the human torque. 
First international congress for the advancement of mechanism, machine, robotics and mechatronics sciences, Beirut,

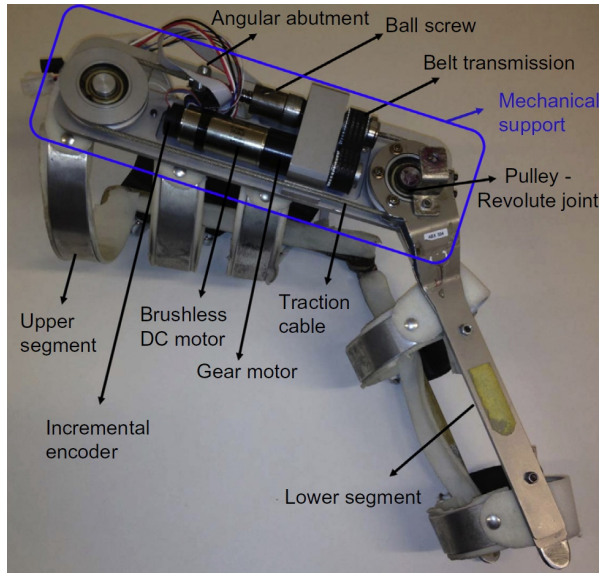

Fig. 1: EICOSI orthosis and its components

Parameters Identification:: The identification of the shank-Orthosis parameters was obtained in [13] by separate identification of each component. A nonlinear least square optimization method was used to find the shank and orthosis parameters. $\theta_{\text {rest }}$ which is the rest position was obtained by means of the incremental encoder then used in the identification process. The Muscular activity of the muscles was monitored through the EMG electrodes. Theses signals are supposed to be null during the rest position. To determine the shank mass and its gravity center, the regression equations of Winter were used considering the subject's weight and height. A passive pendulum test was performed to identify the other parameters. [13] The identified parameters are shown in Table 1 and Table 2. The above parameters were verified using

TABLE I: Orthosis Parameters Identification [13]

\begin{tabular}{cc}
\hline Parameters & Value \pm s.d \\
\hline Inertia $\left(J_{0}\right)$ & $0.0117 \pm 3.5238 \mathrm{~kg} \mathrm{~m}^{2}$ \\
Solid Friction Coefficient $\left(A_{0}\right)$ & $0.3525 \pm 0.2491 \mathrm{~N} \mathrm{~m}$ \\
Viscous Friction Coefficient $\left(B_{0}\right)$ & $0.6928 \pm 0.3811 \mathrm{~N} \mathrm{~m} \mathrm{~s} \mathrm{rad} 1$ \\
Gravity Torque $\left(\tau_{g 0}\right)$ & $0.2424 \pm 0.5518 \mathrm{~N} \mathrm{~m}$ \\
Stiffness $\left(K_{0}\right)$ & $0 \mathrm{~N} \mathrm{~m} \mathrm{\text {rad}^{- }} 1$ \\
\hline
\end{tabular}

TABLE II: Shank Parameters Identification [13]

\begin{tabular}{cc}
\hline Parameters & Value $\pm \mathrm{s.d}$ \\
\hline Inertia $\left(J_{s}\right)$ & $1.5383 \pm 0.7052 \mathrm{~kg} \mathrm{~m}^{2}$ \\
Solid Friction Coefficient $\left(A_{s}\right)$ & $0.3975 \pm 1.3265 \mathrm{~N} \mathrm{~m}$ \\
Viscous Friction Coefficient $\left(B_{s}\right)$ & $4.7528 \pm 2.4557 \mathrm{~N} \mathrm{~m} \mathrm{~s} \mathrm{rad}{ }^{-1}$ \\
Gravity Torque $\left(\tau_{g s}\right)$ & $17.4576 \mathrm{~N} \mathrm{~m}$ \\
Stiffness $\left(K_{s}\right)$ & $0.45 \pm 0.5476 \mathrm{~N} \mathrm{~m} \mathrm{rad} 1$ \\
\hline
\end{tabular}

a cross-validation process. A trajectory was considered and the inverse dynamics of the orthosis were computed then the resulting torque was compared to the measured one when the given trajectory was applied.

\section{EXOSKELETON CONTROL}

The following control laws are tested in order to compare different strategies for the orthosis control:

\section{PID CONTROL}

A standard PID controller, also called the three-term controller, has a transfer function that is generally written in the form given bellow:

$$
G(s)=K_{p}+K_{i} \frac{1}{s}+K_{d} s
$$

Where $K_{p}$ is the proportional gain, $K_{i}$ is the integral gain and $K_{d}$ is the derivative gain. A proportional term for an overall control action proportional to the error signal; an integral term to reduce the steady-state errors, and a derivative term to improve the transient response through high-frequency compensation by a differentiator. For optimum performance $K_{p}, K_{i}$ and $K_{d}$ are mutually dependent in tuning [7].

Stability analysis:: The action torque of a PID controller may be expressed by :

$$
\tau=K_{p} \tilde{\theta}+K_{d} \dot{\tilde{\theta}}+K_{i} \zeta
$$

Where $\tilde{\theta}$ and $\dot{\tilde{\theta}}$ are respectively the errors between current and desired position and velocity, and $\zeta$ is an additional state variable

$$
\begin{gathered}
\dot{\zeta}=\tilde{\theta} \\
\tilde{\theta}=\theta_{d}-\theta \\
\dot{\tilde{\theta}}=\dot{\theta_{d}}-\dot{\theta}
\end{gathered}
$$

We consider a free moving orthosis with $\tau_{h}=0$.

The closed loop equation obtained by substituting $\tau$ may be written as following:

$$
\begin{gathered}
J \ddot{\theta}+\tau_{g} \cos \theta+A \operatorname{Sign} \dot{\theta}+B \dot{\theta}+K\left(\theta-\theta_{\text {rest }}\right) \\
=K_{p} \tilde{\theta}+K_{d} \dot{\tilde{\theta}}+K_{i} \zeta \\
\dot{\zeta}=\tilde{\theta}
\end{gathered}
$$

The unique equilibrium of the system is a constant vector :

$$
\begin{gathered}
\left(\begin{array}{c}
\zeta_{e q} \\
\tilde{\theta}_{e q} \\
\tilde{\tilde{\theta}}_{e q}
\end{array}\right)=\left(\begin{array}{c}
\frac{1}{K_{i}} \tau_{g} \cos \theta_{d} \\
0 \\
0
\end{array}\right) \\
\frac{d}{d t}\left(\begin{array}{c}
\zeta \\
\tilde{\theta} \\
\dot{\tilde{\theta}}
\end{array}\right)=\left(\begin{array}{c}
\tilde{\theta} \\
\dot{\tilde{\theta}} \\
\ddot{\theta_{d}}+\frac{1}{J}\left(K_{p} \tilde{\theta}+K_{d} \dot{\tilde{\theta}}+K_{i} \zeta-\right. \\
\tau_{g} \cos \theta-A \operatorname{Sign} \dot{\theta}-B \dot{\theta}-K\left(\theta-\theta_{\text {rest }}\right)
\end{array}\right)
\end{gathered}
$$

By operating the following change of variables:

$$
\omega=\alpha \zeta-\frac{\alpha}{k_{i}} \tau_{g} \cos \theta_{d}+\tilde{\theta}
$$

With $\alpha>0$ We define the following globally positive definite Lyapunov function:

$$
\begin{gathered}
V(\tilde{\theta}, \dot{\theta}, \omega)=\frac{1}{2}\left[\frac{\omega^{2}}{\alpha} K_{i}+\alpha K_{d} \tilde{\theta}^{2}+J \alpha \dot{\theta}^{2}-2 \dot{\theta} \tilde{\theta} J\right] \\
+\frac{1}{2} \tilde{\theta}^{2}\left[K_{p}-\frac{1}{\alpha} K_{i}\right]+U\left(\theta_{d}-\theta\right)-U\left(\theta_{d}\right)+\tilde{\theta} \tau_{g} \cos \theta_{d}
\end{gathered}
$$

Where $U$ denote the potential energy of the system and

$$
\frac{\partial U}{\partial \theta}=\tau_{g} \cos \theta
$$

$\alpha$ is the positive constant used in the variable chagne in (11) The time derivative of this equation will be :

$$
\begin{aligned}
\dot{V}(\tilde{\theta}, \dot{\theta}, \omega) & =-\dot{\theta}^{2}\left(K_{d}-\alpha J\right)-\tilde{\theta}^{2}\left(\alpha K_{p}-K_{i}\right) \\
& -\alpha \tilde{\theta}\left(\tau_{g} \cos \theta_{d}-\tau_{g} \cos \theta\right)
\end{aligned}
$$

Using the following proprety related to the Gravitational torques of robotic systems:

"For robots, having exclusively revolute joints, there exists a 
number $K_{g}>0$ such as : $K_{g} \geq \frac{\partial g(q)}{\partial q}$ " [14] We assume: $K_{g}=\tau_{g}$ which satisfies the above, and $\alpha$ satisfies the following inequalities:

$$
\frac{K_{d}}{J}>\alpha>\frac{K_{i}}{K_{p}-K_{g}}
$$

and

$$
K_{p}>K_{g}=\tau_{g}
$$

Hense :

$$
\begin{gathered}
-\dot{\theta}^{2}\left(K_{d}-\alpha J\right) \leq 0 \\
-\tilde{\theta}^{2}\left(\alpha K_{p}-K_{i}\right) \leq 0 \\
-\alpha \tilde{\theta}\left(\tau_{g} \cos \theta_{d}-\tau_{g} \cos \theta\right) \leq 0 \quad \forall \theta \in \mathcal{D} \\
\mathcal{D}=\left[-\frac{\pi}{2}, 0\right]
\end{gathered}
$$

It is easily established that, when $\alpha$ is picked to satisfy the condition (15), $V(\tilde{\theta}, \dot{\theta}, \omega)$ is globally positive definite, while the derivative of Lyapunov function (14) is negative semidefinite. If we can find conditions on the gains $K_{p}, K_{i} a n d K_{d}$ of the PID controller such that the origin of the closed-loop (10) is asymptotically stable, at least for sufficiently small values of the initial states $\omega(0), \tilde{\theta}(0)$ and $\dot{\theta}(0)$, and particularly $\tilde{\theta}(t)$; the state tends asymptotically to zero, then we can conclude that at least locally the position control objective is achieved [14].

\section{A. Sliding Mode Control :}

Sliding mode is one of the commonly used methods to control nonlinear systems. Intuitively the approach is based on the remark that it is easier to control a first order system. A time varying surface is defined as following:

$$
s=\left(\frac{d}{d t}+\lambda\right) \tilde{\theta}
$$

With $\lambda$ a positive constant . [15]

Stability Analysis: We consider a free moving orthosis with $\tau_{h}=0$ Considering the following Lyapunov function:

$$
V=\frac{1}{2} s^{2}
$$

To satisfy the condition $\dot{V} \leq 0$, the following control torque is proposed:

$$
\begin{aligned}
\tau= & \tau_{g} \cos \theta+A \operatorname{Sign} \dot{\theta}+B \dot{\theta}+K\left(\theta-\theta_{\text {rest }}\right) \\
& +J\left[-\lambda \dot{\theta}+\ddot{\theta_{d}}+\lambda \dot{\theta_{d}}+K_{\text {slide }} \operatorname{sign}(s)\right]
\end{aligned}
$$

With $K_{\text {slide }}$ a positive gain. The time derivative of the Lyapunov function is :

$$
\begin{gathered}
\dot{V}=s \dot{s}=s(\ddot{\tilde{\theta}}+\lambda \dot{\tilde{\theta}}) \\
=s\left(\ddot{\theta_{d}}-\frac{1}{J}\left[-\tau_{g} \cos \theta-A \operatorname{Sign} \dot{\theta}-B \dot{\theta}-K\left(\theta-\theta_{\text {rest }}\right)+\right.\right. \\
\tau]+\lambda \dot{\tilde{\theta}}
\end{gathered}
$$

By replacing $\tau$ by its expression in 20, we obtain :

$$
\dot{V}=-k_{\text {slide }} \operatorname{Sign}(s)
$$

Since $\operatorname{s.sign}(s) \leq 0 \forall s, \dot{V} \leq 0$ the control torque applied ensure the asymptotic stability of the system.[16]

\section{B. Adaptive Control :}

Inspired by the sliding mode approach [8] [15], this model reference control law takes advantages in computing continually the system parameters to achieve better tracking performances of the desired trajectory. Assumption: the current and desired states up to the third derivative are bounded. The time varying surface is defined as following : $s=(\dot{\tilde{\theta}}+\lambda \tilde{\theta})$ with $\lambda$ a positive scalar The dynamocs of the adaptive law are given:

$$
\begin{array}{cc}
\dot{\hat{J}}=-a_{1}\left(\ddot{\theta_{d}}-\lambda \dot{\tilde{\theta}}\right) s & \dot{\hat{A}}=-a_{2} \operatorname{sign}(\dot{\theta}) s \\
\dot{\hat{B}}=-a_{3} \dot{\theta} s & \dot{\hat{\tau_{g}}}=-a_{4} \cos \theta s \\
\dot{\hat{K}}=-a_{5}\left(\theta-\theta_{\text {rest }}\right) s
\end{array}
$$

With $a_{i}, i \in 1, ., 5$ are positive scaling parameters. Where $\hat{J}, \hat{A}, \hat{B}$ are the estimated system inertia, solid and viscous friction parameters, gravity torque and stiffness. The control torque is given by the following formula

$$
\begin{gathered}
\tau=\hat{J}\left(\ddot{\theta_{d}}-\lambda \dot{\tilde{\theta}}\right)+\hat{A} \operatorname{Sign}(\dot{\theta})+\hat{B} \dot{\theta} \\
+\hat{\tau_{g}} \cos \theta+\hat{K}\left(\theta-\theta_{\text {rest }}\right)-K_{\text {adapt }} s
\end{gathered}
$$

With $K_{\text {adapt }}$ a positive gain.

Stability Analysis: We consider a free moving orthosis $\tau_{h}=0$.The stability of the system when implemented this control law was studied in [8]. We define the positive Lyapunov function:

$$
\begin{aligned}
V= & \frac{1}{2} J s^{2}+\frac{1}{2 a_{1}} \tilde{J}^{2}+\frac{1}{2 a_{2}} \tilde{A}^{2}+\frac{1}{2 a_{3}} \tilde{B}^{2} \\
& +\frac{1}{2 a_{4}}{\tilde{\tau_{g}}}^{2}+\frac{1}{2 a_{5}} \tilde{K}^{2}+K_{\text {adapt }} \tilde{\theta}^{2}
\end{aligned}
$$

Where : $\tilde{J}=J-\hat{J}, \tilde{A}=A-\hat{A}, \tilde{B}=B-\hat{B}, \tilde{\tau_{g}}=\tau_{g}-$ $\tan _{g}, \tilde{K}=K=\hat{K}$ The derivative of this Lyapunov function is :

$$
\begin{gathered}
\dot{V}=s J \dot{s}+\frac{1}{a_{1}} \tilde{J} \dot{\tilde{J}}+\frac{1}{a_{2}} \tilde{A} \dot{\tilde{A}}+\frac{1}{a_{3}} \tilde{B} \dot{\tilde{B}}+\frac{1}{a_{4}} \tilde{\tau_{g}} \dot{\tilde{\tau} g} \\
+\frac{1}{a_{5}} \tilde{K} \dot{\tilde{K}}+2 K_{a d a p t} \lambda \tilde{\theta} \dot{\tilde{\theta}}=-k_{a d a p t} \dot{\tilde{\theta}}^{2}-k_{\text {adapt }} \lambda^{2} \tilde{\theta}^{2} \leq 0
\end{gathered}
$$

All the signals and their derivatives are bounded, hence, the second derivative of the Lyapunov function is bounded which implies that $\mathrm{V}$ is uniformly continuous. The state $(\tilde{\theta}, \dot{\tilde{\theta}})$ asymptotically converges to zero with a domain of attraction equal to $[-\pi, \pi]$.

\section{Simulation Results}

The three control laws are simulated using Matlab/Simulink considering the model of orthosis EICOSI of LISSI-Lab. This control laws are applied to the orthosis in order to track a predefined trajectory taking into account an external human torque. Four scenarios are tested in this simulation.

Scenario 1: The users effort is considered null $\left(\tau_{h}=0\right)$. The control law is tested with a desired trajectory representing flexion and extension movements $\left[-\frac{\pi}{2},-\frac{\pi}{4}\right]$. A white Gaussian noise with null mean and standard deviation of 0.07 N.m is added to the torque $\tau_{h}=0$ in order to simulate real conditions. For this scenario, a particular attention should be paid to the control torques values (Fig.3). For same performances, different torques values are delivered by sliding mode and the adaptive sliding mode controller. These results are a 
First international congress for the advancement of mechanism, machine, robotics and mechatronics sciences, Beirut,

significant indication for the energy consumption. Without adaptive mechanism, sliding mode controller consumes less energy. This aspect could be another parameter to be taken in consideration while designing controllers for such nonlinear systems.

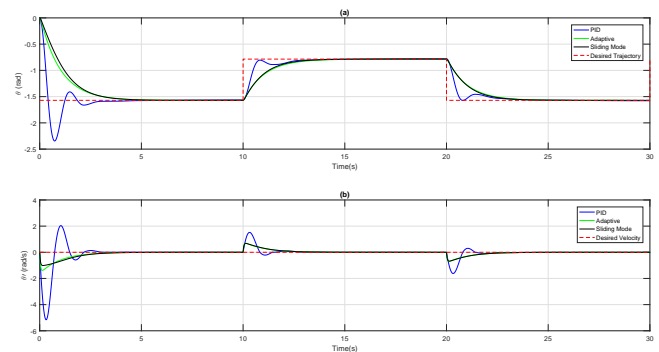

Fig. 2: Flexions and extensions with no human effort: (a) The kneejoint angle. (b) angular velocity
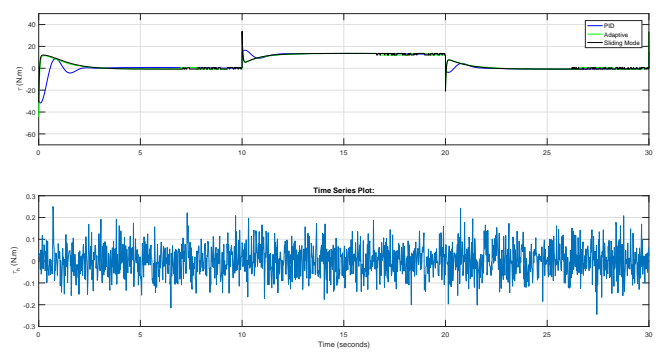

Fig. 3: Flexions and extensions with no human effort: The human and control torques

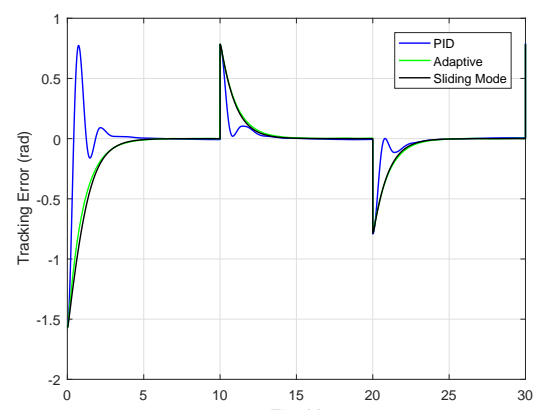

Fig. 4: Flexions and extensions Wime(sth no human effort: Tracking Error

Scenario 2: The user's effort is considered null $\left(\tau_{h}=0\right)$. The control law is tested with a sinusoidal desired trajectory $\theta_{d}=-\frac{1}{4} \sin \left(2 \pi \times 0.2 \times t-\frac{1}{4}\right)$. This simulation put in evidence the inaccuracy of a PID controller. Adding to this, it was proven that the human limb movements yield to variations in the model parameters, that a classical PID doesnt compensate. In [17], the authors have modelized the muscular contraction in a viscous environment by a linear damper element that can be described as following:

$$
\bar{F}_{d}^{m}=\bar{b}_{m} \bar{v}_{m}
$$

Where $\bar{b}_{m}$ is the normalized damping coefficient and $\bar{v}_{m}$ is the normalized contraction velocity. In [18], it was put in evidence that there is a correlation between this velocity and the muscle length during muscular contraction. Several works [19] , [1] have studied the biomechanical properties related to human movement. Because of these properties, more robust control approaches should be developed to deal with uncertainty and disturbances specific to systems evolving human movement.

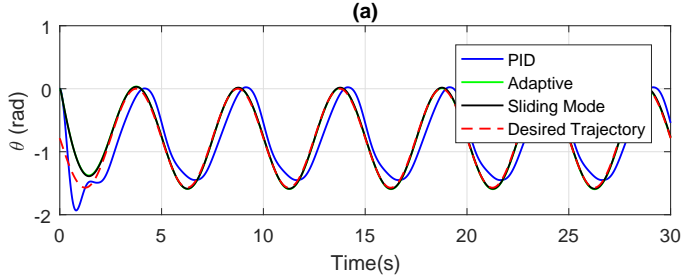

(b)

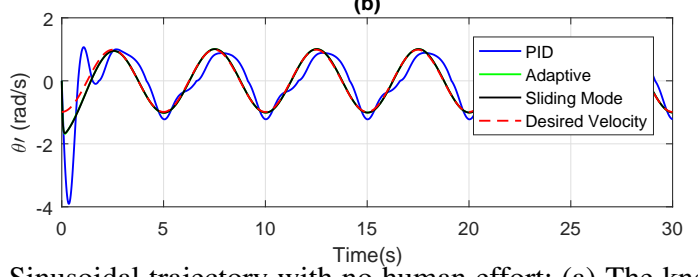

Fig. 5: Sinusoidal trajectory with no human effort: (a) The knee-joint angle. (b) The knee-joint angular velocity
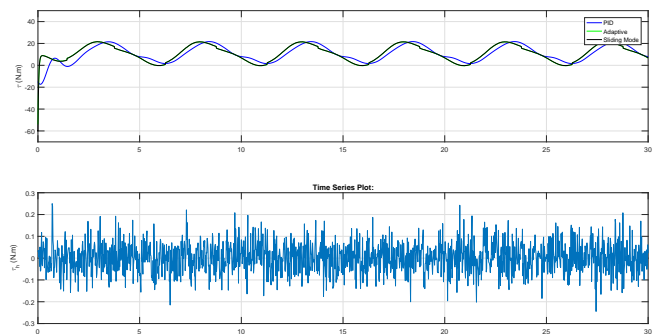

Fig. 6: Sinusoidal trajectory with no human effort: The human and control torques

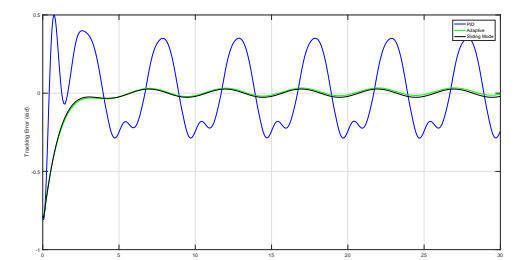

Fig. 7: Sinusoidal trajectory with no human effort: Tracking Error

Scenario 3: The wearer develops an effort to ensure the desired movement $\left(\tau_{h} \neq 0\right)$. A sinusoidal signal $\tau_{h}=$ $4 \times \sin (2 \pi \times 0.2 \times t)$ is added to represent the assisting human effort. The user applies at $\mathrm{t}=10 \mathrm{~s}$ an assisting torque for $20 \mathrm{~s}$. At $t=30 \mathrm{~s}$, the user stops his effort and the orthosis is driven only by the actuators torque. The control law is tested with a sinusoidal desired trajectory $\theta_{d}=-\frac{1}{4} \sin \left(2 \pi \times 0.2 \times t-\frac{1}{4}\right)$. The main motivation of this scenario is to observe the control values and the trajectory tracking performances when an assistive human effort is added in an Assistance As Needed context. The control laws are compared in terms of trajectory tracking accuracy and the additional control torque delivered. Fig.8 shows the added human torque results in a small overshoot of the knee-joint angle. For the adaptive controller, the tracking errors were decreasing during the period when the human torque was applied. This is due to the parameters adaptation. A better regulation can be obtained by increasing the parameters convergence gain $a_{i}$. 
First international congress for the advancement of mechanism, machine, robotics and mechatronics sciences, Beirut,
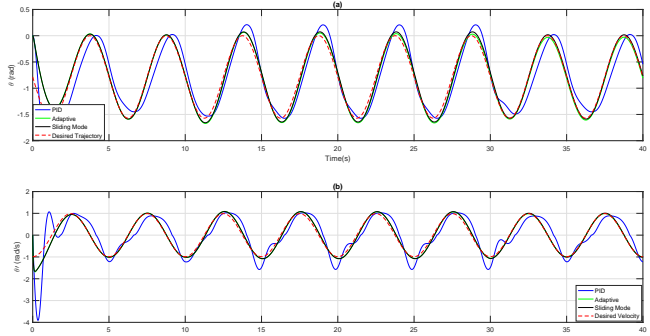

Fig. 8: Sinusoidal trajectory with assisting human effort:(a) The kneejoint angle. (b)The knee-joint angular velocity
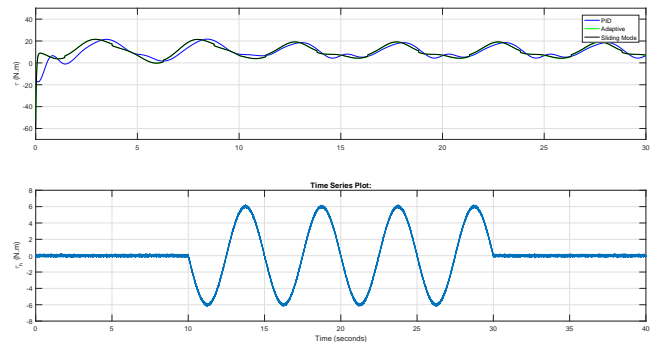

Fig. 9: Sinusoidal trajectory with assisting human effort: The human and control torques

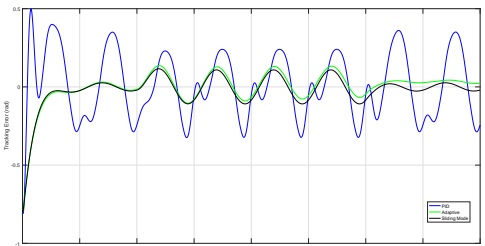

Fig. 10: Sinusoidal trajectory with assisting human effort: Tracking error

Scenario 4: In this scenario, we consider a resistive subject who develops an effort from $\mathrm{t}=10 \mathrm{~s}$ till $\mathrm{t}=30 \mathrm{~s}$, that is opposing to the orthosis movement. A sinusoidal signal $\tau_{h}=4 \times \sin (2 \pi \times 0.2 \times t)$ is added to represent the resistive human effort. The user applies it at $\mathrm{t}=10 \mathrm{~s}$ for $20 \mathrm{~s}$. At $\mathrm{t}=30 \mathrm{~s}$, the user stops his resistance and the orthosis is driven only by the actuators torque. The control law is tested with a sinusoidal desired trajectory: $\theta_{d}=-\frac{1}{4} \sin \left(2 \pi \times 0.2 \times t-\frac{1}{4}\right)$. This scenario considers the resistive rehabilitation case. The results show that the Sliding mode controller has better tracking performances in terms of tracking errors. The adaptive controller tends to decrease these errors by adapting the parameters to ensure the better performances.

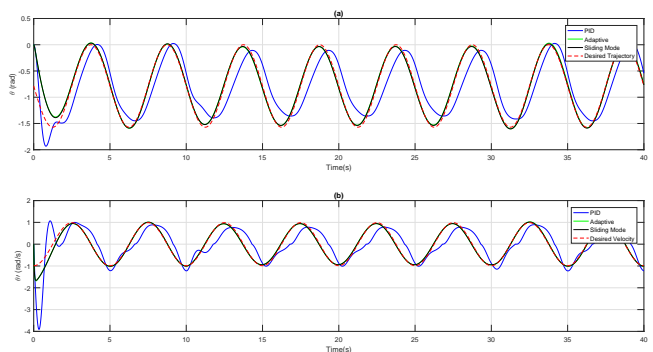

Fig. 11: Sinusoidal trajectory with resisting human effort: (a) The knee-joint angle. (b) The knee-joint angular velocity
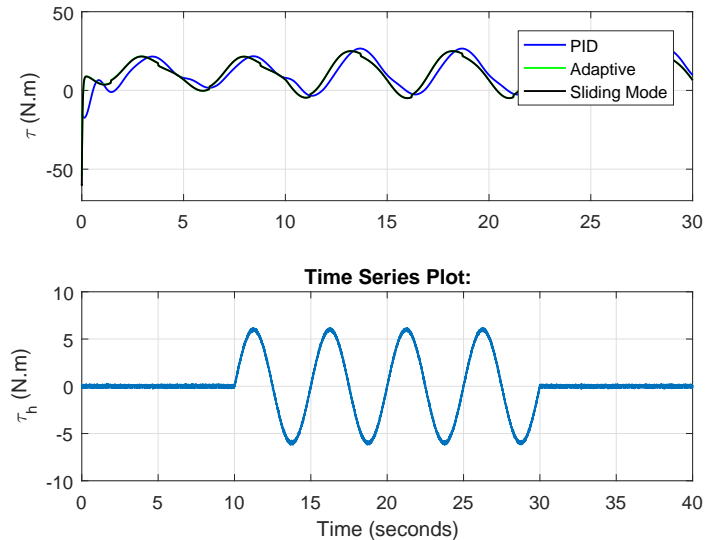

Fig. 12: Sinusoidal trajectory with resisting human effort: The human and control torques

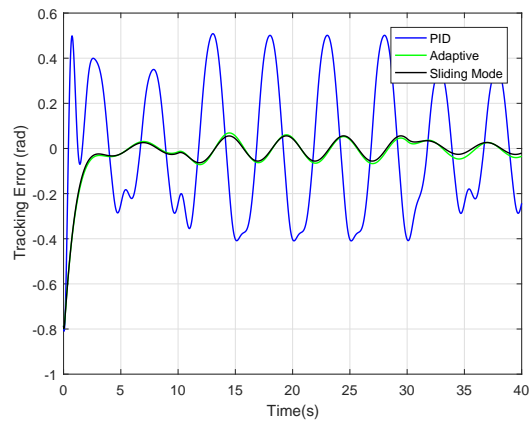

Fig. 13: Sinusoidal trajectory with resisting human effort: Tracking error

Scenario 5: We consider the second scenario conditions (sinusoidal trajectory). The purpose of this scenario is to evaluate the robustness of the controllers with regard to parameters uncertainties. Bias of $20 \%, 40 \%, 80 \%$ of parameters values were introduced to the model parameters. The simulation results show that parameters errors introduce a fixed tracking error. This error is consequently reduced by the adaptation mechanism with the adaptive control Fig.16. Fig. 17 Shows how the error is reduced during the adaptation process. The parameters adapt to ensure a better convergence of the trajectory

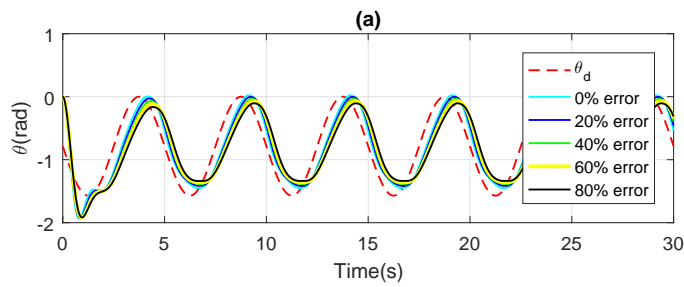

(b)

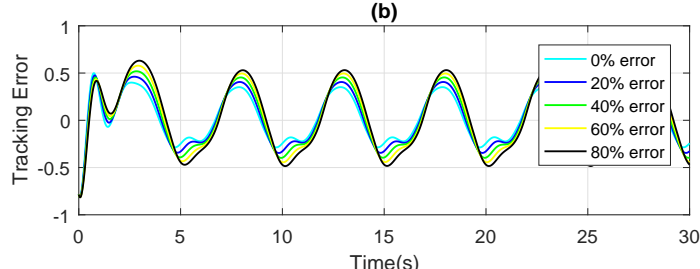

Fig. 14: PID Robustness Tests: The Knee Joint angle and Tracking Error 
First international congress for the advancement of mechanism, machine, robotics and mechatronics sciences, Beirut, Lebanon, October 2017
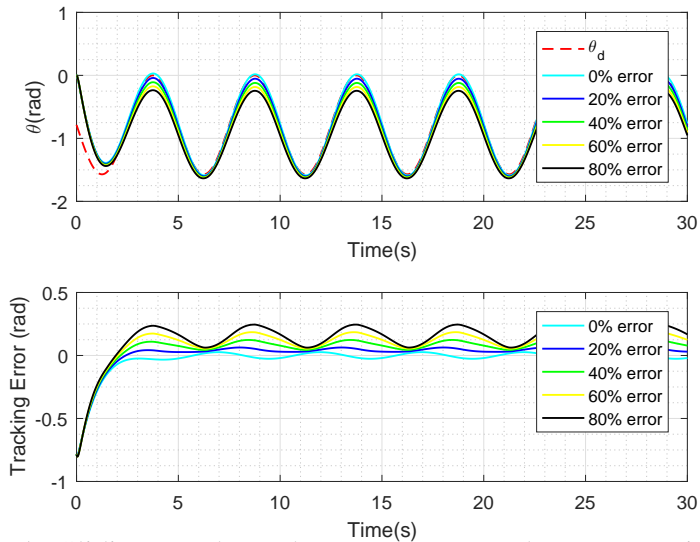

Fig. 15: Sliding mode Robustness Tests: The Knee Joint angle Tracking Error

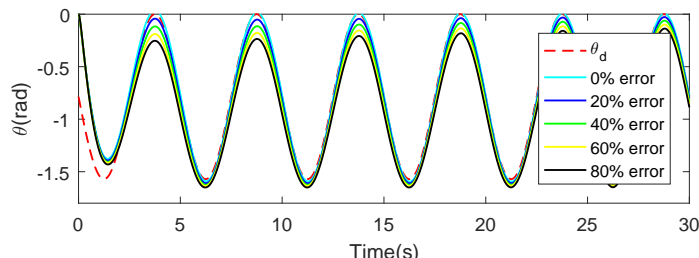

(c)

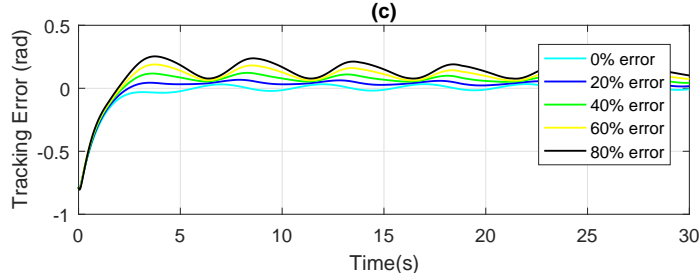

Fig. 16: Adaptive Control Robustness Tests: The Knee Joint angle Tracking Error

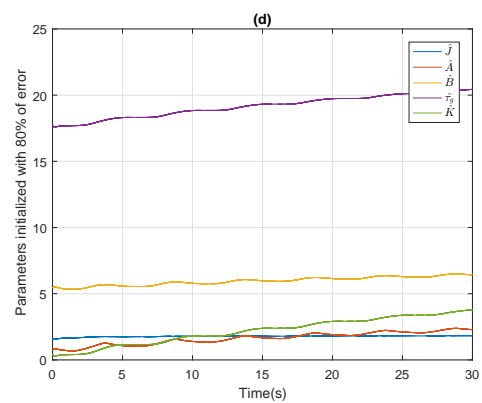

Fig. 17: Adaptive Control Robustness Tests: Parameters Adaptation

\section{CONCLUSiOn AND Future Work}

The main objective of this study is to bring out the benefit of orienting control strategies towards adaptive approach for human assistance and rehabilitation processes to compensate for changes in the human-orthosis dynamics. Three different control laws were tested in different operational scenarios covering three study cases which are : passive rehabilitation, assistance as needed and resistive rehabilitation contexts, a robustness test with regards to parameters uncertainties was performed on the three controllers. The simulation results confirmed that an additional adaptive model based terms including some of the system dynamics can compensate for uncertainties, disturbances and for dynamic changes due to human activity. Future experiments may consolidate those results and demonstrate the relevance of such approaches. This work can be further improved and enriched with real experimentations on human assistance wearable robot.

\section{REFERENCES}

[1] C. M. Jansen, J. E. Windau, P. M. Bonutti, and M. V. Brillhart, "Treatment of a knee contracture using a knee orthosis incorporating stress-relaxation techniques," Physical therapy, vol. 76, no. 2, pp. 182$186,1996$.

[2] C. Rudhe, U. Albisser, M. L. Starkey, A. Curt, and M. Bolliger, "Reliability of movement workspace measurements in a passive arm orthosis used in spinal cord injury rehabilitation," Journal of neuroengineering and rehabilitation, vol. 9, no. 1, p. 37, 2012.

[3] L. Marchal-Crespo and D. J. Reinkensmeyer, "Review of control strategies for robotic movement training after neurologic injury," Journal of neuroengineering and rehabilitation, vol. 6, no. 1, p. 20, 2009.

[4] T. S. Buchanan, D. G. Lloyd, K. Manal, and T. F. Besier, "Neuromusculoskeletal modeling: estimation of muscle forces and joint moments and movements from measurements of neural command," Journal of applied biomechanics, vol. 20, no. 4, pp. 367-395, 2004.

[5] C. Fleischer and G. Hommel, "A human-exoskeleton interface utilizing electromyography," IEEE Transactions on Robotics, vol. 24, no. 4, pp. 872-882, 2008

[6] D. P. Ferris and C. L. Lewis, "Robotic lower limb exoskeletons using proportional myoelectric control," in Engineering in Medicine and Biology Society, 2009. EMBC 2009. Annual International Conference of the IEEE, pp. 2119-2124, IEEE, 2009.

[7] K. H. Ang, G. Chong, and Y. Li, "Pid control system analysis, design, and technology," IEEE transactions on control systems technology, vol. 13 , no. 4 , pp. $559-576,2005$.

[8] H. Rifai, S. Mohammed, B. Daachi, and Y. Amirat, "Adaptive control of a human-driven knee joint orthosis," in Robotics and Automation (ICRA), 2012 IEEE International Conference on, pp. 2486-2491, IEEE, 2012.

[9] J. A. Blaya and H. Herr, "Adaptive control of a variable-impedance ankle-foot orthosis to assist drop-foot gait," IEEE Transactions on neural systems and rehabilitation engineering, vol. 12, no. 1, pp. 24-31, 2004.

[10] J. W. Noble and S. D. Prentice, "Adaptation to unilateral change in lower limb mechanical properties during human walking," Experimental brain research, vol. 169, no. 4, pp. 482-495, 2006.

[11] P.-C. Kao and D. P. Ferris, "Motor adaptation during dorsiflexionassisted walking with a powered orthosis," Gait \& posture, vol. 29 , no. 2, pp. 230-236, 2009.

[12] H. Rifaï, M. B. Abdessalem, A. Chemori, S. Mohammed, and Y. Amirat, "Augmented- 1 adaptive control of an actuated knee joint exoskeleton: From design to real-time experiments," in Robotics and Automation (ICRA), 2016 IEEE International Conference on, pp. 5708-5714, IEEE, 2016.

[13] H. Rifaï, S. Mohammed, W. Hassani, and Y. Amirat, "Nested saturation based control of an actuated knee joint orthosis," Mechatronics, vol. 23, no. 8, pp. 1141-1149, 2013.

[14] R. Kelly, V. S. Davila, and J. A. L. Perez, Control of robot manipulators in joint space. Springer Science \& Business Media, 2006.

[15] J.-J. E. Slotine and W. Li, "On the adaptive control of robot manipulators," The international journal of robotics research, vol. 6, no. 3, pp. 49-59, 1987.

[16] M. Boualbani and A.Bousri, "Commande avancée d'un exosquelette," Master's thesis, Université de Montpellier 2, Montpellier, France, 2016.

[17] D. G. Thelen et al., "Adjustment of muscle mechanics model parameters to simulate dynamic contractions in older adults," TransactionsAmerican Society Of Mechanical Engineers Journal Of Biomechanical Engineering, vol. 125, no. 1, pp. 70-77, 2003.

[18] A. Hill, "The heat of shortening and the dynamic constants of muscle," Proceedings of the Royal Society of London B: Biological Sciences, vol. 126, no. 843, pp. 136-195, 1938.

[19] F. E. Zajac, "Muscle and tendon properties models scaling and application to biomechanics and motor," Critical reviews in biomedical engineering, vol. 17, no. 4, pp. 359-411, 1989. 